\title{
A rare case of maxillary nodular fasciitis: Case report and short literature review
}

\section{Mauro Massarelli ｜ Francesco Paparo (i) | Simone Benedetti}

Department of Maxillo-Facial Surgery, Azienda Ospedaliera S.Maria, Terni, Italy

\section{Correspondence}

Francesco Paparo, Department of MaxilloFacial Surgery, Azienda Ospedaliera S.Maria, Terni, Italy.

Email: dottorpaparo@gmail.com

\begin{abstract}
Nodular fasciitis has to be considered as an option in the presence of a solitary, rapidly growing mass even in older adults. When possible, direct surgery should be preferred to FNAC, particularly in the older adults because of high misdiagnostic risk.
\end{abstract}

\section{K E Y W O R D S}

elderly, FNAC, head, neck, nodular fasciitis, pseudosarcomatous fibromatosis

\section{$1 \quad$ INTRODUCTION}

Nodular fasciitis (NF) is an unusual, benign, rapidly growing, and contained condition which rarely affects the head and neck district. NF affects people regardless of gender or race, and it is more frequent in young adults. It is often confused with malignancies, especially in the elderly patients. Nowadays, histopathology and immunohistochemistry play a pivotal role in diagnosis as fine-needle aspiration cytology (FNAC) can be correctly diagnosed only if the cytologist is aware of its clinical and cytologic features. Following a short Literature review, the authors report a rare case of maxillary nodular fasciitis in an 85-year-old patient, pointing out that in chosen cases, direct surgery could avoid misdiagnosis, delays and overtreatment.

Nodular fasciitis (NF) or pseudosarcomatous fibromatosis was first described in $1955,{ }^{1}$ and it is a rare benign condition in which fibroblasts rapidly proliferate from the deep fascia to the adjacent tissues, including muscle and subcutaneous tissues. It is a contained, solitary lesion whose pathogenesis is still uncertain. NF seems to be related to local traumas in only $10 \%-15 \%$ of cases. ${ }^{2}$

In most of the nontraumatic cases, recent studies identified rearrangements of the USP6 gene, often with MYH9. ${ }^{3}$ In about half of the patients, local tenderness or pain is reported whereas paresthesia and shooting pain are mostly present when NF involves the upper limbs. Given the self-limiting nature of the lesion, the definition of "transient neoplasia" has been proposed. ${ }^{3} \mathrm{NF}$ shows no predilection for specific gender or race, and affects the upper extremities (43\%), the trunk (25\%), and head and neck district $(10 \%)$; first symptoms often appear in young adults aged 20-40 years. ${ }^{2}$ Differential diagnosis encloses fibrosarcoma, fibroma, schwannoma, ${ }^{4,5}$ fibrous histiocytoma, ossifying lipoma, ${ }^{6}$ and desmoid lesions. Histopathology and immunohistochemistry play a pivotal role in diagnosis as FNAC might be useful but not conclusive. ${ }^{7-10}$ In fact, NF can be correctly diagnosed only if the cytologist is aware of its clinical and cytologic features. ${ }^{11}$ After proper surgical excision, recurrence is very rare. ${ }^{2}$

Surgical techniques may vary depending on the volume and location of the mass. Many reconstructive options are available such as local flaps, pedicled flaps, and microvascular flaps. Special care must be used to preserve facial nerve integrity. In case of damage or mass involvement, facial reanimation is indicated. ${ }^{5,11,12}$

\section{CASE PRESENTATION}

A 85-year-old female with type II diabetes and heart failure, treated with metformin, antihypertensives and 
antiplatelet medicaments, presented to our department with a 4-month history of a rapidly growing mass, located within the soft tissues of the right cheek. The lesion was indolent, and no other signs or symptoms were further reported.

Clinical history did not highlight any local recent event. Patient examination revealed a palpable, round, painless, and firm lesion located on the right cheek, without alteration of the overlying skin.

An US scan confirmed the presence of a 25-mm nodular mass adherent to the fascia without local neck lymph node involvement.

Soon after, the patient underwent complete surgical excision of the lesion under local anesthesia which was easily obtained by a small intraoral access. Definitive histopathological examination of the surgical specimen showed atypical fibrocyte and histiocyte proliferation in a fibromyxoid stroma. The cells resulted negative for the presence of S-100, cytokeratin AE1-AE3, and desmin. Given these features, diagnosis of nodular fasciitis (NF) was made.

At two-year follow-up, there was no local recurrence.

\section{3 | DISCUSSION AND CONCLUSIONS}

Nodular fasciitis is a rare growing contained benign condition. It is more frequent in the young adults, it rapidly proliferates and spreads to adjacent tissues, thus mimicking a malignancy. It is most commonly misdiagnosed as spindle cell sarcoma, owing to its rapid growth, high cellularity, cellular, and nuclear polymorphism. ${ }^{8}$ A recent study ${ }^{13}$ reported that $18 \%$ of cases were misdiagnosed as sarcoma. Moreover, Plaza et al reported that two-third of their cases had been misdiagnosed as sarcoma which is more frequent in late adult life, like in our patient. ${ }^{14}$

In this case, the lesion was easily approachable and the patient had a poor compliance due to her age and not fair general condition.

That is why it was decided to intervene directly throughout local surgery instead of performing FNAC because it would have led to either diagnostic delay or misdiagnosis with consequent overtreatment.

In conclusion, although rare, nodular fasciitis has to be considered as an option in the presence of a solitary, rapidly growing mass even in elderly adults. In our case, clinical history would have suggested a malignancy, but the result was a benign lesion.

To the purpose, we believe that, when possible, direct surgery should be preferred to FNAC, particularly in late adult patients thus avoiding misdiagnosis, delays, and overtreatment.

\section{ACKNOWLEDGMENTS}

We thank the anonymous referees for their useful suggestions. Published with written consent of the patient.

\section{CONFLICT OF INTEREST}

None declared.

\section{AUTHOR CONTRIBUTIONS}

MM: made substantial contribution to conception, design and acquisition of data analysis, and interpretation of data. PF: has been involved in drafting the manuscript and revising it critically for important intellectual content. BS: has been involved in drafting the manuscript and revising it critically for important intellectual content.

\section{ETHICAL APPROVAL}

All procedures performed in this study were in accordance with the ethical standards of the institutional and national research committee and with the 1964 Helsinki declaration and its later amendments or comparable ethical standards.

\section{ORCID}

Francesco Paparo (iD https://orcid. org/0000-0003-3388-1601

\section{REFERENCES}

1. Be K, Keasbey L, Kaplan L. Subcutaneous pseudosarcomatous fibromatosis (fasciitis). Am J Clin Pathol. 1955;25(3):241-252.

2. Shibata Y, Yanaba K, Ito K, Nishimura R, Miyawaki T, Nakagawa H. Nodular fasciitis on the face. J Dermatol. 2016;43(10):1235-1236.

3. Baranov E, Hornick JL. Soft tissue special issue: fibroblastic and myofibroblastic neoplasms of the head and neck. Head Neck Pathol. 2020;14(1):43-58.

4. Aboh IV, Chisci G, Cascino F, et al. Giant palatal schwannoma. $J$ Craniofac Surg. 2014;25(5):e418-e420.

5. Gennaro P, Nastro Siniscalchi E, Gabriele G, Cascone P. Trigeminal and facial schwannoma: a case load and review of the literature. Eur Rev Med Pharmacol Sci. 2012;16(suppl 4):8-12.

6. Aboh IV, Chisci G, Salini C, et al. Submandibular ossifying lipoma. J Craniofac Surg. 2015;26(3):973-974.

7. Allison DB, Wakely PE Jr, Siddiqui MT, Ali SZ. Nodular fasciitis: A frequent diagnostic pitfall on fine-needle aspiration. Cancer Cytopathol. 2017;125(1):20-29.

8. Magro G. Differential Diagnosis of Benign Spindle Cell Lesions. Surg Pathol Clin. 2018;11(1):91-121.

9. Gan S, Xie D, Dai H, et al. Proliferative myositis and nodular fasciitis: a retrospective study with clinicopathologic and radiologic correlation. Int J Clin Exp Pathol. 2019;12(12):4319-4328.

10. Wang XL, De Schepper AM, Vanhoenacker F, et al. Nodular fasciitis: correlation of MRI findings and histopathology. Skeletal Radiol. 2002;31(3):155-161.

11. Gennaro P, Gabriele G, Aboh IV, et al. The Second Division of Trigeminal Nerve for Corneal Neurotization: A Novel One-Stage Technique in Combination With Facial Reanimation. J Craniofac Surg. 2019;30(4):1252-1254 
12. Gennaro P, Chisci G, Gabriele G, Iannetti G. Conservative surgical and microsurgical techniques for the management of dental implants that impinge on the inferior alveolar nerve. $\mathrm{Br} \mathrm{J}$ Oral Maxillofac Surg. 2014;52(6):566-568.

13. Rani D, Gupta A. Cytological diagnosis and misdiagnosis of nodular fasciitis. J Cytol. 2019;36(4):196-199.

14. Plaza JA, Mayerson J, Wakely PE. Nodular fasciitis of the hand: A potential diagnostic pitfall in fine-needle aspiration cytopathology. Am J Clin Pathol. 2005;123:388-393.
How to cite this article: Massarelli M, Paparo F, Benedetti S. A rare case of maxillary nodular fasciitis: Case report and short literature review. Clin Case Rep. 2021;9:31-33. https://doi.org/10.1002/ccr3.3377 